\title{
Chemical Constituents from the Whole Plant of Cuscuta reflexa
}

\author{
Tin Thu Thu Aung ${ }^{1,3} \cdot$ Meng-Yuan Xia ${ }^{1} \cdot$ Pyae Phyo Hein ${ }^{1,3} \cdot$ Rong Tang $^{1} \cdot$ Dong-Dong Zhang ${ }^{1,4} \cdot$ Jun Yang $^{1,4}$. \\ Xue-Fei Yang ${ }^{1,4}$. Dong-Bao Hu ${ }^{2} \cdot$ Yue-Hu Wang ${ }^{1,4}$
}

Received: 26 June 2020 / Accepted: 9 September 2020 / Published online: 21 September 2020

(c) The Author(s) 2020

\begin{abstract}
Two new $2 H$-pyran-2-one glucosides, cuscutarosides A (1) and B (2), and one new steroidal glucoside, $7 \beta$-methoxy- $\beta$ sitosterol 3-O- $\beta$-glucopyranoside (3), together with 12 known compounds (4-15) were isolated from the whole plant of Cuscuta reflexa (Convolvulaceae) collected from Myanmar. The chemical structures of these new compounds were elucidated based on extensive spectroscopic analysis. The antiobesity activity of these isolates was evaluated using porcine pancreatic lipase (PPL), and the antiplatelet aggregation activity was screened using rabbit platelets induced by thrombin, plateletactivating factor (PAF), arachidonate (AA), or collagen. $7 \beta$-Methoxy- $\beta$-sitosterol 3-O- $\beta$-glucopyranoside (3) showed weak PPL inhibitory activity. Cuscutaroside A (1), its acetylated derivative (1a), and scrophenoside B (8) showed weak inhibitory activity against rabbit platelet aggregation induced by collagen. Compound 1a also showed inhibitory activity against rabbit platelet aggregation induced by AA.
\end{abstract}

\section{Graphic Abstract}
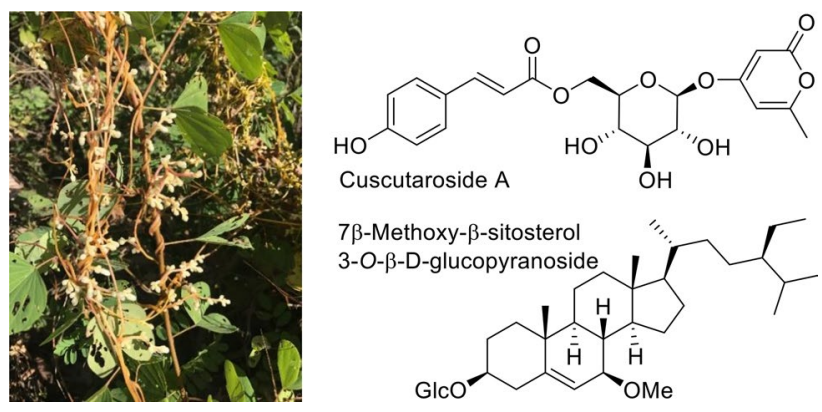

Keywords Cuscuta reflexa $\cdot$ Porcine pancreatic lipase $\cdot$ Platelet aggregation $\cdot 2 H$-pyran-2-one glucosides $\cdot$ Steroidal glucosides

Tin Thu Thu Aung and Meng-Yuan Xia contributed equally to this work.

Electronic supplementary material The online version of this article (https://doi.org/10.1007/s13659-020-00265-x) contains supplementary material, which is available to authorized users.

Dong-Bao Hu

lh@yxnu.edu.cn

Yue-Hu Wang

wangyuehu@mail.kib.ac.cn

1 Key Laboratory of Economic Plants and Biotechnology and the Yunnan Key Laboratory for Wild Plant Resources, Kunming Institute of Botany, Chinese Academy of Sciences, Kunming 650201, People's Republic of China
2 School of Chemical Biology and Environment, Yuxi Normal University, Yuxi 653100, People's Republic of China

3 University of Chinese Academy of Sciences, Beijing 100049, People's Republic of China

4 Southeast Asia Biodiversity Research Institute, Chinese Academy of Sciences, Yezin, Nay Pyi Taw 05282, Myanmar 


\section{Introduction}

Cuscuta reflexa Roxb. (Convolvulaceae), a twining parasitic plant, is distributed in China, Afghanistan, India, Indonesia, Malaysia, Myanmar, Nepal, Pakistan, Sri Lanka, and Thailand [1]. In Nujiang Prefecture, Yunnan Province, China, the Lisu people call it mu-gua-zhua and use its whole plant or seeds to treat soreness and weakness of the waist and knees, erectile dysfunction, spermatorrhea, diabetes, dizziness, hypopsia, and threatened abortion [2]. Named shwe-new or shwe-nwe-pin (Hsay) in Myanmar, the whole plant of $C$. reflexa is used to treat inflammation, irregularities of the blood, and other diseases [3]. It is also medically used in India, Nepal, Bangladesh, and Pakistan [4]. Based on published studies, the major chemical constituents of $C$. reflexa include flavonoids, coumarins, phenylpropanoids, triterpenoids, and cardiac glycosides [4-7].

A previous study showed that extracts of $C$. reflexa possess an antiobesity effect [8], but no further studies clarified the active compounds responsible for the activity. Pancreatic lipase inhibition is one of the most widely studied mechanisms for antiobesity treatment [9]. Purified human lipase (HPL) and porcine pancreatic lipase (PPL) show similar specific activities [10]. Recently, the extract of $C$. reflexa was found to have in vitro thrombolytic activity [11]. This finding may somewhat explain its traditional use in the treatment of irregularities of the blood. However, no active constituents were reported in this research. In the current study, we report the structural elucidation of three new compounds from the whole plant of $C$. reflexa and the results of a bioassay for the inhibitory activities against PPL and rabbit platelet aggregation.

\section{Results and Discussion}

\subsection{Structural Elucidation}

Through chromatographic techniques, three new compounds (1-3, Fig. 1) and 12 known compounds (4-15, Supplementary Material, Fig. S1) were obtained from the EtOH extract of C. reflexa.

Based on ${ }^{13} \mathrm{C}$ NMR data (Table 1 ) and the HRESIMS ion peak at $m / z 457.1106[\mathrm{M}+\mathrm{Na}]^{+}$(calcd for $\mathrm{C}_{21} \mathrm{H}_{22} \mathrm{NaO}_{10}$, 457.1111), the molecular formula of 1 was deduced to be $\mathrm{C}_{21} \mathrm{H}_{22} \mathrm{O}_{10}$. The ${ }^{1} \mathrm{H}$ and ${ }^{13} \mathrm{C}$ NMR data (Table 1) indicated the presence of a $p$-coumaroyl moiety $\left[\delta_{\mathrm{H}} 7.60\right.$ $(1 \mathrm{H}, \mathrm{d}, J=15.9 \mathrm{~Hz}), 7.48(2 \mathrm{H}$, br d, $J=8.7 \mathrm{~Hz}), 6.79$ $(2 \mathrm{H}$, br d, $J=8.7 \mathrm{~Hz})$, and $6.36(1 \mathrm{H}, \mathrm{d}, J=15.9 \mathrm{~Hz}) ; \delta_{\mathrm{C}}$ 169.0], one methyl group $\left[\delta_{\mathrm{H}} 2.20(3 \mathrm{H}, \mathrm{s}) ; \delta_{\mathrm{C}} 19.8\right]$, and one $\beta$-glucopyranosyl group $\left[\delta_{\mathrm{H}} 5.05(\mathrm{~d}, J=7.4 \mathrm{~Hz})\right]$. By comparing its NMR data with those of $4-(\beta-\mathrm{D}-$ glucopyranosyloxy)-6-methyl-2H-pyran-2-one (5) [12], it was implied that compound $\mathbf{1}$ might be a $p$-coumaroyl derivative of 4-glucopyranosyloxy-6-methyl- $2 \mathrm{H}$-pyran2 -one. Based on the 2D NMR spectra of $\mathbf{1}$, especially the HMBC correlations from $\mathrm{H}-1^{\prime}$ to $\mathrm{C}-4$ and $\mathrm{H}_{2}-6^{\prime}$ to $\mathrm{C}-9^{\prime \prime}$, the 4-glucopyranosyloxy-6-methyl-2H-pyran-2-one moiety was confirmed, and the $p$-coumaroyl group was located at $6^{\prime}-\mathrm{OH}$ through an ester bond. An acetylated derivative (1a) was obtained using pyridine and acetic anhydride. We tried to obtain a crystal of $\mathbf{1 a}$, but unsuccessful. After acidic hydrolysis of 1, D-glucose was obtained. Thus, the chemical structure of 1 was determined as shown in Fig. 1 and named cuscutaroside A.

The molecular formula of cuscutaroside B (2) was determined to be $\mathrm{C}_{17} \mathrm{H}_{24} \mathrm{O}_{9}$ based on the ${ }^{13} \mathrm{C}$ NMR data (Table 1) and the HRESIMS ion peak at $m / z, 395.1313[\mathrm{M}+\mathrm{Na}]^{+}$

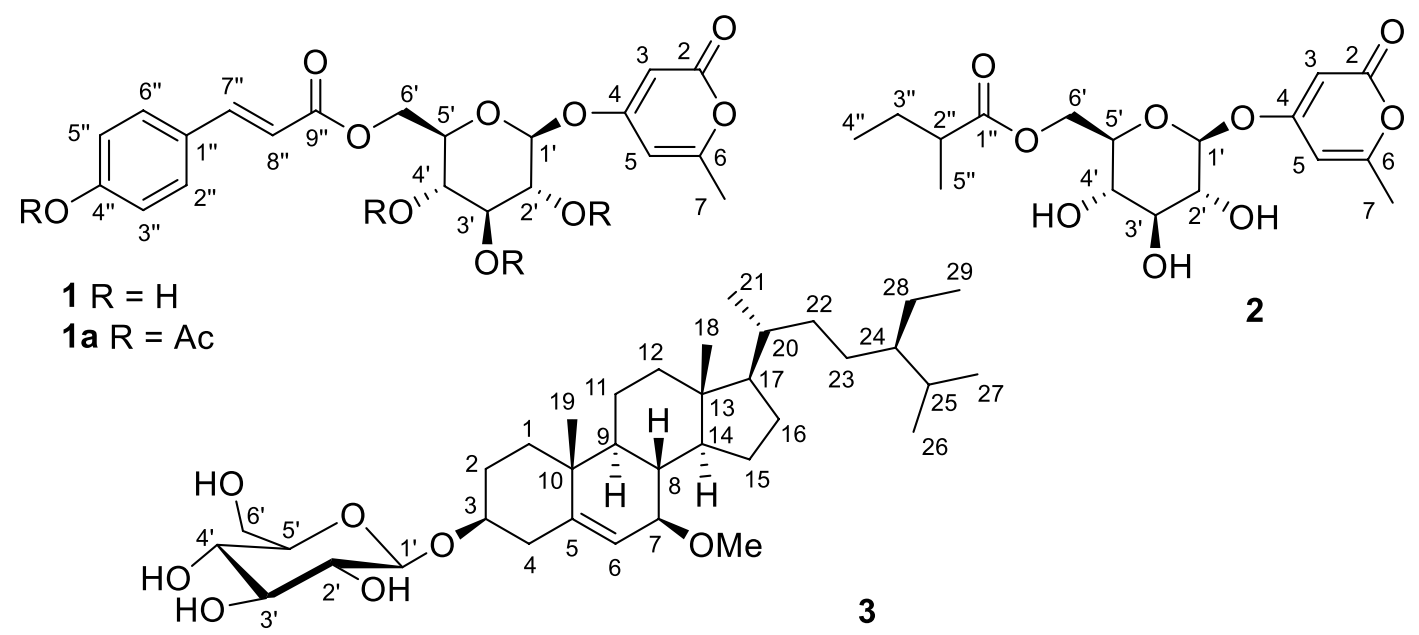

Fig. 1 Chemical structures of new compounds (1-3) and the acetylated derivative of 1 
Table $1{ }^{1} \mathrm{H}$ and ${ }^{13} \mathrm{C}$ NMR data of $\mathbf{1}$ and $\mathbf{2}$ in methanol- $d_{4}(\delta$ in ppm, $J$ in $\mathrm{Hz}$ )

\begin{tabular}{|c|c|c|c|c|}
\hline \multirow[t]{2}{*}{ No. } & \multicolumn{2}{|l|}{1} & \multicolumn{2}{|l|}{2} \\
\hline & $\delta_{\mathrm{H}}(500 \mathrm{MHz})$ & $\delta_{\mathrm{C}}(125 \mathrm{MHz})$ & $\delta_{\mathrm{H}}(600 \mathrm{MHz})$ & $\delta_{\mathrm{C}}(150 \mathrm{MHz})$ \\
\hline 2 & & 167.4, C & & 167.2, C \\
\hline 3 & $5.73(\mathrm{~d}, 2.0)$ & $91.9, \mathrm{CH}$ & $5.66(\mathrm{~d}, 2.1)$ & $91.6, \mathrm{CH}$ \\
\hline 4 & & 171.3, C & & $171.3, \mathrm{C}$ \\
\hline 5 & $6.08(\mathrm{~m})$ & 101.5, CH & $6.10(\mathrm{~m})$ & $101.5, \mathrm{CH}$ \\
\hline 6 & & $164.8, \mathrm{C}$ & & $164.8, \mathrm{C}$ \\
\hline 7 & $2.20(\mathrm{~s})$ & $19.8, \mathrm{CH}_{3}$ & $2.25(\mathrm{~s})$ & 19.7, $\mathrm{CH}_{3}$ \\
\hline $1^{\prime}$ & $5.05(\mathrm{~d}, 7.4)$ & $100.7, \mathrm{CH}$ & $5.05(\mathrm{~d}, 7.5)$ & $100.6, \mathrm{CH}$ \\
\hline $2^{\prime}$ & $3.48(\mathrm{~m})$ & $74.3, \mathrm{CH}$ & $3.43(\mathrm{dd}, 9.3,7.5)$ & $74.3, \mathrm{CH}$ \\
\hline $3^{\prime}$ & $3.49(\mathrm{~m})$ & $77.7, \mathrm{CH}$ & $3.47(\mathrm{dd}, 9.3,8.7)$ & $77.5, \mathrm{CH}$ \\
\hline $4^{\prime}$ & $3.39(\mathrm{dd}, 9.4,8.9)$ & $71.5, \mathrm{CH}$ & $3.33(\mathrm{dd}, 9.7,8.7)$ & $71.5, \mathrm{CH}$ \\
\hline $5^{\prime}$ & $3.78(\mathrm{~m})$ & $76.0, \mathrm{CH}$ & $3.73(\mathrm{~m})$ & $75.8, \mathrm{CH}$ \\
\hline $6^{\prime}$ & $\begin{array}{l}4.55(\mathrm{dd}, 12.0,2.0) \\
4.24(\mathrm{dd}, 12.0,7.3)\end{array}$ & 64.6, $\mathrm{CH}_{2}$ & $\begin{array}{l}4.43(\mathrm{dd}, 11.9,2.1) \\
4.17(\mathrm{dd}, 11.9,7.1)\end{array}$ & $64.5, \mathrm{CH}_{2}$ \\
\hline $1^{\prime \prime}$ & & 127.1, C & & $178.2, \mathrm{C}$ \\
\hline $2^{\prime \prime}$ & 7.48 (br d, 8.7) & $131.4, \mathrm{CH}$ & $2.43(\mathrm{~m})$ & $42.3, \mathrm{CH}$ \\
\hline $3 "$ & $6.79(\mathrm{br} \mathrm{d}, 8.7)$ & $116.9, \mathrm{CH}$ & $\begin{array}{l}1.65(\mathrm{~m}) \\
1.47(\mathrm{~m})\end{array}$ & 27.9, $\mathrm{CH}_{2}$ \\
\hline $4 "$ & & 161.5, C & $0.97(\mathrm{t}, 7.3)$ & $11.9, \mathrm{CH}_{3}$ \\
\hline $5^{\prime \prime}$ & 6.79 (br d, 8.7) & $116.9, \mathrm{CH}$ & $1.13(\mathrm{~d}, 7.0)$ & 17.0, $\mathrm{CH}_{3}$ \\
\hline $6^{\prime \prime}$ & 7.48 (br d, 8.7) & $131.4, \mathrm{CH}$ & & \\
\hline $7^{\prime \prime}$ & $7.60(d, 15.9)$ & $147.0, \mathrm{CH}$ & & \\
\hline $8^{\prime \prime}$ & $6.36(\mathrm{~d}, 15.9)$ & 114.7, $\mathrm{CH}$ & & \\
\hline $9^{\prime \prime}$ & & $169.0, \mathrm{C}$ & & \\
\hline
\end{tabular}

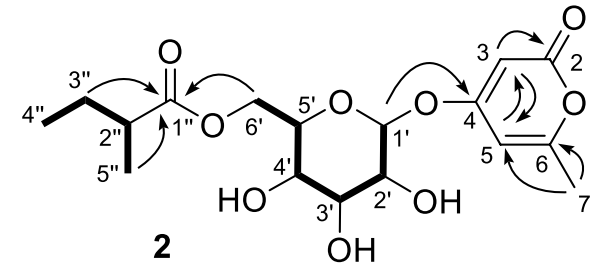

2

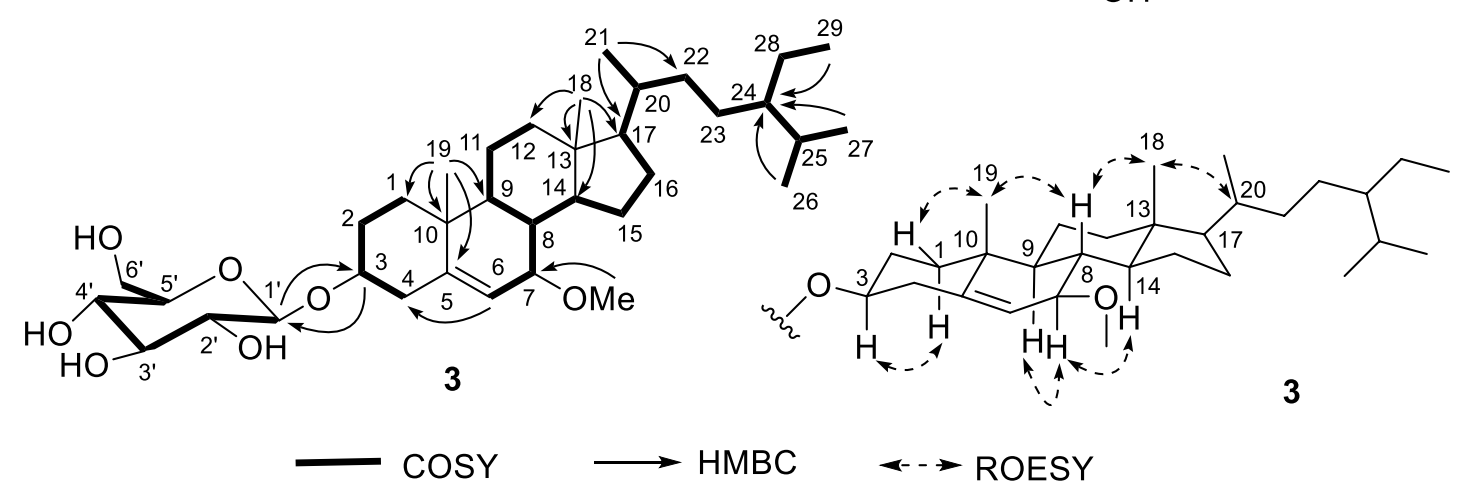

Fig. 2 Key 2D NMR correlations of compounds 1-3

(calcd for $\mathrm{C}_{17} \mathrm{H}_{24} \mathrm{NaO}_{9}, 395.1318$ ). The ${ }^{1} \mathrm{H}$ and ${ }^{13} \mathrm{C}$ NMR data of 2 (Table 1) indicated the presence of one 6-methyl$2 H$-pyran-2-one moiety $\left(\delta_{\mathrm{C}} 171.3,167.2,164.8,101.5\right.$, and 91.6) [12], one $\beta$-glucopyranosyl group $\left[\delta_{\mathrm{H}} 5.05(\mathrm{~d}\right.$,
$J=7.5 \mathrm{~Hz})$ ], and one 2-methylbutyryl group $\left(\delta_{\mathrm{C}} 178.2,42.3\right.$, 27.9, 17.0, and 11.9) [13]. Compound 2 was deduced as a 2-methylbutyryl derivative of 4-( $\beta$-glucopyranosyloxy)6-methyl-2H-pyran-2-one by the COSY and HMBC 
Table $2{ }^{1} \mathrm{H}(800 \mathrm{MHz})$ and ${ }^{13} \mathrm{C}$ (200 MHz) NMR data of $\mathbf{3}$ and 4 in methanol- $d_{4}(\delta$ in ppm, $J$ in $\mathrm{Hz}$ )

\begin{tabular}{|c|c|c|c|c|}
\hline \multirow[t]{2}{*}{ No. } & \multicolumn{2}{|l|}{3} & \multicolumn{2}{|l|}{4} \\
\hline & $\overline{\delta_{\mathrm{H}}}$ & $\delta_{\mathrm{C}}$ & $\overline{\delta_{\mathrm{H}}}$ & $\delta_{\mathrm{C}}$ \\
\hline $\begin{array}{l}1 \beta \\
1 \alpha\end{array}$ & $\begin{array}{l}1.88(\mathrm{~m}) \\
1.05(\mathrm{~m})\end{array}$ & $38.2, \mathrm{CH}_{2}$ & $\begin{array}{l}2.00(\mathrm{~m}) \\
1.25(\mathrm{~m})\end{array}$ & $39.9, \mathrm{CH}_{2}$ \\
\hline 2 & $\begin{array}{l}1.96(\mathrm{~m}) \\
1.63(\mathrm{~m})\end{array}$ & $30.7, \mathrm{CH}_{2}$ & $\begin{array}{l}2.07(\mathrm{~m}) \\
1.73(\mathrm{~m})\end{array}$ & $30.4, \mathrm{CH}_{2}$ \\
\hline 3 & $3.62(\mathrm{~m})$ & $79.4, \mathrm{CH}$ & $3.76(\mathrm{~m})$ & $78.5, \mathrm{CH}$ \\
\hline $\begin{array}{l}4 \alpha \\
4 \beta\end{array}$ & $\begin{array}{l}2.50(\mathrm{ddd}, 13.3,4.7,2.1) \\
2.30(\mathrm{br} \mathrm{t}, 13.3)\end{array}$ & $39.4, \mathrm{CH}_{2}$ & $\begin{array}{l}2.68(\mathrm{~m}) \\
2.47(\mathrm{ddd}, 13.5,11.8,2.2)\end{array}$ & 39.6, $\mathrm{CH}_{2}$ \\
\hline 5 & & 145.7, C & & $168.8, \mathrm{C}$ \\
\hline 6 & $5.48(\mathrm{t}, 1.7)$ & $122.5, \mathrm{CH}$ & $5.69(\mathrm{~d}, 1.2)$ & $126.5, \mathrm{CH}$ \\
\hline 7 & $3.44(\mathrm{dt}, 8.5,2.0)$ & $83.4, \mathrm{CH}$ & & 204.7, C \\
\hline 8 & $1.52(\mathrm{~m})$ & $38.3, \mathrm{CH}$ & $2.32(\mathrm{dd}, 12.7,10.9)$ & $46.6, \mathrm{CH}$ \\
\hline 9 & $1.05(\mathrm{~m})$ & $49.9, \mathrm{CH}$ & $1.52(\mathrm{~m})$ & $51.5, \mathrm{CH}$ \\
\hline 10 & & $37.8, \mathrm{C}$ & & $37.4, \mathrm{C}$ \\
\hline 11 & $\begin{array}{l}1.52(\mathrm{~m}) \\
1.40(\mathrm{~m})\end{array}$ & $22.3, \mathrm{CH}_{2}$ & $1.64(\mathrm{~m})$ & 22.3, $\mathrm{CH}_{2}$ \\
\hline $\begin{array}{l}12 \beta \\
12 \alpha\end{array}$ & $\begin{array}{l}2.04(\mathrm{dt}, 12.8,3.8) \\
1.16(\mathrm{~m})\end{array}$ & 41.0, $\mathrm{CH}_{2}$ & $\begin{array}{l}2.08(\mathrm{~m}) \\
1.16(\mathrm{~m})\end{array}$ & $40.1, \mathrm{CH}_{2}$ \\
\hline 13 & & $44.0, \mathrm{C}$ & & $44.3, \mathrm{C}$ \\
\hline 14 & $1.16(\mathrm{~m})$ & $57.7, \mathrm{CH}$ & $1.32(\mathrm{~m})$ & $51.5, \mathrm{CH}$ \\
\hline 15 & $\begin{array}{l}1.71(\mathrm{~m}) \\
1.40(\mathrm{~m})\end{array}$ & $26.8, \mathrm{CH}_{2}$ & $1.26(\mathrm{~m})$ & 27.4, $\mathrm{CH}_{2}$ \\
\hline 16 & $\begin{array}{l}1.88(\mathrm{~m}) \\
1.29(\mathrm{~m})\end{array}$ & 29.6, $\mathrm{CH}_{2}$ & $\begin{array}{l}1.89(\mathrm{~m}) \\
1.30(\mathrm{~m})\end{array}$ & 29.6, $\mathrm{CH}_{2}$ \\
\hline 17 & $1.11 \mathrm{~m}$ & $56.9, \mathrm{CH}$ & $1.12(\mathrm{~m})$ & $56.1, \mathrm{CH}$ \\
\hline 18 & $0.72(\mathrm{~s})$ & $12.3, \mathrm{CH}_{3}$ & $0.73(\mathrm{~s})$ & $12.3, \mathrm{CH}_{3}$ \\
\hline 19 & $1.07(\mathrm{~s})$ & $19.4, \mathrm{CH}_{3}$ & $1.25(\mathrm{~s})$ & 17.7, $\mathrm{CH}_{3}$ \\
\hline 20 & $1.38(\mathrm{~m})$ & $37.4, \mathrm{CH}$ & $1.38(\mathrm{~m})$ & $37.5, \mathrm{CH}$ \\
\hline 21 & $0.96(\mathrm{~d}, 6.5)$ & $19.4, \mathrm{CH}_{3}$ & $0.97(\mathrm{~d}, 6.6)$ & $19.5, \mathrm{CH}_{3}$ \\
\hline 22 & $\begin{array}{l}1.38(\mathrm{~m}) \\
1.05(\mathrm{~m})\end{array}$ & $35.1, \mathrm{CH}_{2}$ & $\begin{array}{l}1.38(\mathrm{~m}) \\
1.05(\mathrm{~m})\end{array}$ & $35.1, \mathrm{CH}_{2}$ \\
\hline 23 & $1.21(\mathrm{~m})$ & 27.2, $\mathrm{CH}_{2}$ & $1.22(\mathrm{~m})$ & 27.2, $\mathrm{CH}_{2}$ \\
\hline 24 & $0.95(\mathrm{~m})$ & $47.3, \mathrm{CH}$ & $0.96(\mathrm{~m})$ & $47.3, \mathrm{CH}$ \\
\hline 25 & $1.69(\mathrm{~m})$ & $30.4, \mathrm{CH}$ & $1.68(\mathrm{~m})$ & $30.4, \mathrm{CH}$ \\
\hline 26 & $0.84(\mathrm{~d}, 6.9)$ & $19.4, \mathrm{CH}_{3}$ & $0.84(\mathrm{~d}, 6.9)$ & $19.4, \mathrm{CH}_{3}$ \\
\hline 27 & $0.88(\mathrm{~d}, 7.2)$ & $20.2, \mathrm{CH}_{3}$ & $0.87(\mathrm{~d}, 7.2)$ & $20.2, \mathrm{CH}_{3}$ \\
\hline 28 & $1.33(\mathrm{~m})$ & $24.2, \mathrm{CH}_{2}$ & $1.32(\mathrm{~m})$ & $24.2, \mathrm{CH}_{2}$ \\
\hline 29 & $0.87(\mathrm{t}, 7.0)$ & $12.3, \mathrm{CH}_{3}$ & $0.90(\mathrm{t}, 7.1)$ & $12.4, \mathrm{CH}_{3}$ \\
\hline $1^{\prime}$ & $4.39(\mathrm{~d}, 7.8)$ & $102.5, \mathrm{CH}$ & $4.40(\mathrm{~d}, 7.8)$ & $102.7, \mathrm{CH}$ \\
\hline $2^{\prime}$ & $3.15(\mathrm{dd}, 9.1,7.8)$ & $75.1, \mathrm{CH}$ & $3.15(\mathrm{dd}, 9.0,7.8)$ & $75.1, \mathrm{CH}$ \\
\hline $3^{\prime}$ & $3.36(\mathrm{~m})$ & $78.1, \mathrm{CH}$ & $3.36(\mathrm{dd}, 9.0,8.6)$ & $78.1, \mathrm{CH}$ \\
\hline $4^{\prime}$ & $3.27(\mathrm{~m})$ & 71.7, $\mathrm{CH}$ & $3.27(\mathrm{~m})$ & 71.7, $\mathrm{CH}$ \\
\hline $5^{\prime}$ & $3.26(\mathrm{~m})$ & 77.9, $\mathrm{CH}$ & $3.27(\mathrm{~m})$ & 78.0, $\mathrm{CH}$ \\
\hline $6^{\prime}$ & $\begin{array}{l}3.85(\mathrm{dd}, 11.7,1.5) \\
3.65(\mathrm{dd}, 11.7,5.3)\end{array}$ & $62.8, \mathrm{CH}_{2}$ & $\begin{array}{l}3.86(\mathrm{dd}, 11.9,1.7) \\
3.65(\mathrm{dd}, 11.9,5.4)\end{array}$ & $62.8, \mathrm{CH}_{2}$ \\
\hline 7-OMe & $3.28(\mathrm{~s})$ & $54.9, \mathrm{CH}_{3}$ & & \\
\hline
\end{tabular}

correlations (Fig. 2). Based on the HMBC correlations from $\mathrm{H}_{2}-6^{\prime}$ to $\mathrm{C}-1^{\prime \prime}$, the 2-methylbutyryl group was located at 6 '-OH. Thus, the structure of 2 (cuscutaroside B) was elucidated to be 4-[ $\beta$-6- $O$-(2-methylbutyryl)-glucopyranosyloxy]-
6-methyl-2H-pyran-2-one. We were unable to calculate the ECD spectrum of 2 . The absolute configuration of C-2" remains unknown. 
Based on ${ }^{13} \mathrm{C}$ NMR (Table 2) and HRESIMS data, compound 3 was determined to have the molecular formula $\mathrm{C}_{36} \mathrm{H}_{62} \mathrm{O}_{7}$. The ${ }^{1} \mathrm{H}$ and ${ }^{13} \mathrm{C}$ NMR data (Table 2) of 3 indicated the presence of one $\beta$-glucopyranosyl group $\left[\delta_{\mathrm{H}} 4.39\right.$ $(\mathrm{d}, J=7.8 \mathrm{~Hz})]$, one methoxy group $\left[\delta_{\mathrm{H}} 3.28(3 \mathrm{H}, \mathrm{s}) ; \delta_{\mathrm{C}} 54.9\right]$, six methyl groups $\left[\delta_{\mathrm{H}} 1.07(3 \mathrm{H}, \mathrm{s}), 0.96(3 \mathrm{H}, \mathrm{d}, J=6.5 \mathrm{~Hz})\right.$, $0.84(3 \mathrm{H}, \mathrm{d}, J=6.9 \mathrm{~Hz}), 0.88(3 \mathrm{H}, \mathrm{d}, J=7.2 \mathrm{~Hz}), 0.87(3 \mathrm{H}, \mathrm{t}$, $J=7.0 \mathrm{~Hz})$, and $0.72(3 \mathrm{H}, \mathrm{s})]$, and one trisubstituted double bond $\left[\delta_{\mathrm{H}} 5.48(\mathrm{t}, J=1.7 \mathrm{~Hz}) ; \delta_{\mathrm{C}} 145.7\right.$ and 122.5$]$. Comparison of its NMR data with those of 7-oxo- $\beta$-sitosterol 3-O- $\beta$ D-glucopyranoside (4, Table 2$)$ and $7 \beta$-hydroxysitosterol-3$O-\beta$-D-glucopyranoside indicated that compound 3 might be a 7-oxygenated derivative of daucosterol [14-16], which was confirmed by the COSY and HMBC correlations (Fig. 2). According to the HMBC correlations from the OMe group to $\mathrm{C}-7, \mathrm{H}-3$ to $\mathrm{C}-1^{\prime}$, and $\mathrm{H}-1^{\prime}$ to $\mathrm{C}-3$, the methoxy group and the glucopyranosyloxy group were located at C-7 and C-3, respectively. The relative configuration of compound $\mathbf{3}$ was partially determined by ROESY correlations (Fig. 2) and coupling constants (Table 2). ROESY correlations of $\mathrm{H}-1 / \beta / \mathrm{H}_{3}$ $19, \mathrm{H}_{3}-19 / \mathrm{H}-8, \mathrm{H}-8 / \mathrm{H}_{3}-18$, and $\mathrm{H}_{3}-18 / \mathrm{H}-20$ were observed, indicating that these protons are cofacial, and thus, $\mathrm{H}-1 \alpha$ and $\mathrm{H}-17$ are $\alpha$-oriented. $\mathrm{H}-7$ and $\mathrm{H}-8$ should be in a trans axial relationship because of a large coupling constant for $\mathrm{H}-7 / \mathrm{H}-8$ $(J=8.5 \mathrm{~Hz})$, and thus, $\mathrm{H}-7$ is $\alpha$-oriented. The ROESY correlations of $\mathrm{H}-1 \alpha / \mathrm{H}-3, \mathrm{H}-9 / \mathrm{H}-7$, and $\mathrm{H}-7 / \mathrm{H}-14$ indicated that $\mathrm{H}-3$, $\mathrm{H}-9$, and $\mathrm{H}-14$ are also $\alpha$-oriented. The configurations of C-20 and C-24 on the side chain could not be determined by the ROESY correlations. Because the NMR data of the side chain were highly consistent with those in the literature [14-17], the configurations of C-20 and C-24 were suggested to be the same as those in daucosterol. Finally, compound $\mathbf{3}$ was elucidated to be $7 \beta$-methoxy- $\beta$-sitosterol 3- $O-\beta$-glucopyranoside.

The NMR data of 7 -oxo- $\beta$-sitosterol $3-O-\beta$-Dglucopyranoside (4) in pyridine- $d_{5}$ and $\mathrm{CDCl}_{3}$ have been reported previously $[14,15]$. Its NMR data in methanol$d_{4}$ are presented in Table 2 . The other known compounds, 4-( $\beta$-D-glucopyranosyloxy)-6-methyl-2H-pyran-2-one (5) [12], 4-hydroxyacetophenone (6) [18], piceoside (7) [19], scrophenoside B (8) [20], methyl 4,5-di- $O$-caffeoylquinate (9) [21], methyl 3,5-di- $O$-caffeoylquinate (10) [21], methyl 3,4-di- $O$-caffeoylquinate (11) [21], $(6 S, 9 R)$-roseoside (12) [22], methyl trans-p-hydroxycinnamate (13) [23], ethyl trans-p-hydroxycinnamate (14) [24], and $\mathrm{N}$-trans-feruloyltyramine (15) [25], were determined by comparing their NMR data with those in the literature.

\subsection{Porcine Pancreatic Lipase and Platelet Aggregation Inhibition Assay}

Compounds 1-15 were evaluated for their inhibitory activity against PPL. 7 $\beta$-Methoxysitosterol 3-O- $\beta$ glucopyranoside (3) showed weak PPL inhibitory activity
$\left(\mathrm{IC}_{50}=67.2 \pm 1.7 \mu \mathrm{g} / \mathrm{mL}\right)$ compared with the positive control orlistat $\left(\mathrm{IC}_{50}=0.40 \pm 0.02 \mathrm{ng} / \mathrm{mL}\right)$. 7-Oxo- $\beta$-sitosterol 3-O- $\beta$-D-glucopyranoside (4) showed $12 \pm 2 \%$ inhibition at a concentration of $100 \mu \mathrm{g} / \mathrm{mL}$. The other tested compounds were inactive, with inhibition values less than $10 \%$ at a concentration of $100 \mu \mathrm{g} / \mathrm{mL}$.

Compounds 1-15 and 1a were also evaluated for their inhibitory activity against rabbit platelet aggregation induced by thrombin $(1 \mathrm{U} / \mathrm{mL})$, platelet-activating factor (PAF, $0.4 \mu \mathrm{g} / \mathrm{mL}$ ), arachidonate (AA, $0.5 \mathrm{mM}$ ), or collagen $(4 \mu \mathrm{g} / \mathrm{mL})$. Cuscutaroside A (1), 1a, and scrophenoside B (8) showed weak inhibitory activity against rabbit platelet aggregation induced by collagen with $\mathrm{IC}_{50}$ values of $291.4 \pm 47.9 \mu \mathrm{g} / \mathrm{mL}, 63.8 \pm 4.4 \mu \mathrm{g} / \mathrm{mL}$, and $180.5 \pm 6.7 \mu \mathrm{g} / \mathrm{mL}$, respectively, compared with aspirin $\left(\mathrm{IC}_{50}=33.3 \pm 1.3 \mu \mathrm{g} / \mathrm{mL}\right)$. Compound 1a also showed inhibitory activity against rabbit platelet aggregation induced by AA with an $\mathrm{IC}_{50}$ value of $72.6 \pm 10.5 \mu \mathrm{g} / \mathrm{mL}$ compared with aspirin (inhibition $88.1 \pm 1.1 \%$ at $40 \mu \mathrm{g} / \mathrm{mL}$ ). The other tested compounds were inactive $\left(\mathrm{IC}_{50}>300 \mu \mathrm{g} / \mathrm{mL}\right)$.

\section{Experimental Section}

\subsection{General Experimental Procedures}

Optical rotations were recorded using a JASCO P-1020 Polarimeter (Jasco Corp., Tokyo, Japan). UV spectra were obtained using a Shimadzu UV-2401 PC spectrophotometer (Shimadzu, Kyoto, Japan). ECD spectra were recorded on a Chirascan CD spectrometer (Applied Photophysics Ltd., Leatherhead, UK). ${ }^{1} \mathrm{H}$ and ${ }^{13} \mathrm{C}$ NMR spectra were collected on DRX-500, Avance III-600, and Ascend ${ }^{\mathrm{TM}} 800 \mathrm{MHz}$ spectrometers (Bruker Corp., Karlsruhe, Germany) with TMS as an internal standard. ESIMS and HRESIMS analyses were performed on an API QSTAR Pulsar 1 spectrometer (Applied Biosystems/MDS Sciex, Foster City, CA, USA). EIMS and HREIMS were performed on a Waters AutoSpec Premier p776 spectrometer (Waters, Milford, MA, USA). Silica gel G (80-100 and 300-400 mesh, Qingdao Meigao Chemical Co., Ltd., Qingdao, China), $\mathrm{C}_{18}$ silica gel (40-75 $\mu \mathrm{m}$, Fuji Silysia Chemical Ltd., Aichi, Japan), and Sephadex LH-20 (GE Healthcare Bio-Sciences AB, Uppsala, Sweden) were used for column chromatography, and silica gel $\mathrm{GF}_{254}$ (Qingdao Meigao Chemical Co., Ltd.) on precoated plates was used for preparative thin layer chromatography (TLC). TLC spots were visualized under UV light at $254 \mathrm{~nm}$ and by dipping them into $5 \% \mathrm{H}_{2} \mathrm{SO}_{4}$ in alcohol followed by heating. Semipreparative high-performance liquid chromatography (HPLC) was performed with an Agilent 1200 series pump (Agilent Technologies, Santa Clara, USA) equipped with a diode array detector and an Agilent Zorbax 
SB- $\mathrm{C}_{18}$ column $(5.0 \mu \mathrm{m}, \phi 9.4 \times 250 \mathrm{~mm})$ and a Welch Ultimate $\mathrm{AQ}-\mathrm{C}_{18}$ column $(5.0 \mu \mathrm{m}, \phi 4.6 \times 300 \mathrm{~mm})$.

\subsection{Plant Material}

The plant material, growing on a Bauhinia plant, was collected near Golden Cave $\left(20^{\circ} 55^{\prime} 44.25^{\prime \prime} \mathrm{N}\right.$ and $96^{\circ} 38^{\prime}$ $57.41^{\prime \prime}$ E) in Pindaya Township, Southern Shan State, Myanmar, in December 2016. It was identified as Cuscuta reflexa Roxb. by Dr. Jie Cai and Ms. Jun Yang at the Kunming Institute of Botany, Chinese Academy of Sciences. A voucher specimen (no. MMR631) was deposited at the Southeast Asia Biodiversity Research Institute, Chinese Academy of Sciences, Yezin, Nay Pyi Taw, Myanmar and a copy was placed at the Key Laboratory of Economic Plants and Biotechnology, Kunming Institute of Botany, Chinese Academy of Sciences, China.

\subsection{Extraction and Isolation}

The air-dried powdered whole plant of Cuscuta reflexa $(3.3 \mathrm{~kg})$ was ultrasonically extracted for $30 \mathrm{~min}$ with $70 \%$ EtOH at $60{ }^{\circ} \mathrm{C}$. The EtOH extract $(519.0 \mathrm{~g})$ was suspended in $\mathrm{H}_{2} \mathrm{O}$ and further partitioned with petroleum ether, EtOAc, and $n$-butanol to the yield petroleum ether-soluble portion (6.4 g, A), the EtOAc-soluble portion $(66.8 \mathrm{~g}, \mathrm{~B})$, and the n-butanol soluble portion (276.6 g, C), respectively.

Part B was subjected to column chromatography (silica gel; $\mathrm{CH}_{2} \mathrm{Cl}_{2}-\mathrm{MeOH}, 50: 1 \rightarrow 0: 1$, v/v) to yield four fractions (B1-B4). Fraction B1 was separated on an $\mathrm{RP}_{-} \mathrm{C}_{18}$ silica gel column eluted with $\mathrm{MeOH}-\mathrm{H}_{2} \mathrm{O}(10 \% \rightarrow 100 \%)$ to yield three major subfractions. The $20 \% \mathrm{MeOH}$-eluted portion was purified by Sephadex LH-20 column chromatography $(\mathrm{MeOH})$ and silica gel column chromatography (petroleum ether-EtOAc, 10:1) to yield 6 (8.9 mg). The 40\% MeOHeluted portion was purified by column chromatography (Sephadex LH-20, MeOH), preparative TLC (petroleum ether-acetone, 2:1), and semipreparative HPLC [Agilent Zorbax SB-C ${ }_{18}$ column, MeCN-H ${ }_{2} \mathrm{O}$ (containing $0.05 \% \mathrm{TFA}$ ), $35: 65,2 \mathrm{~mL} / \mathrm{min}]$ to yield $\mathbf{1 3}\left(5.0 \mathrm{mg}, t_{\mathrm{R}}=19.388 \mathrm{~min}\right)$ and $14\left(2.0 \mathrm{mg}, t_{\mathrm{R}}=32.159 \mathrm{~min}\right)$.

Fraction B2 was separated on an $\mathrm{RP}-\mathrm{C}_{18}$ silica gel column eluted with $\mathrm{MeOH}-\mathrm{H}_{2} \mathrm{O}(10 \% \rightarrow 100 \%)$ to yield one main subfraction. The $30 \% \mathrm{MeOH}-$ eluted portion was isolated by Sephadex LH-20 (MeOH) and silica gel column chromatography $\left(\mathrm{CH}_{2} \mathrm{Cl}_{2}-\mathrm{MeOH}, 30: 1\right)$ and further purified by semipreparative HPLC [Welch Ultimate AQ- $\mathrm{C}_{18}$ column, $\mathrm{MeCN}-\mathrm{H}_{2} \mathrm{O}$ (containing 0.05\% TFA), 20:80, $\left.1 \mathrm{~mL} / \mathrm{min}\right]$ to yield 15 (19.4 $\left.\mathrm{mg}, t_{\mathrm{R}}=36.402 \mathrm{~min}\right)$.

Fraction $\mathrm{B} 3$ was separated on an $\mathrm{RP}-\mathrm{C}_{18}$ silica gel column eluted with $\mathrm{MeOH}-\mathrm{H}_{2} \mathrm{O}(10 \% \rightarrow 100 \%)$ to yield two main subfractions (B3-1 and B3-2). B3-1 (40\% MeOH-eluted portion) was separated by silica gel column chromatography $\left(\mathrm{CH}_{2} \mathrm{Cl}_{2}-\mathrm{MeOH}, 100: 1\right.$ and 50:1) to yield B3-1-1 and B3-1-2. B3-1-1 was separated by Sephadex LH-20 (MeOH) and silica gel column chromatography $\left(\mathrm{CH}_{2} \mathrm{Cl}_{2}-\mathrm{MeOH}, 3: 1\right)$ followed by semipreparative HPLC (Agilent Zorbax SB-C 18 column, $\mathrm{MeOH}-\mathrm{H}_{2} \mathrm{O}, 40: 60,2 \mathrm{~mL} / \mathrm{min}$ ) to yield 2 (3.0 $\mathrm{mg}$, $\left.t_{\mathrm{R}}=24.052 \mathrm{~min}\right)$ and $8\left(2.1 \mathrm{mg}, t_{\mathrm{R}}=37.642 \mathrm{~min}\right) . \mathrm{B} 3-1-2$ was separated by Sephadex LH-20 (MeOH) and silica gel column chromatography $\left(\mathrm{CH}_{2} \mathrm{Cl}_{2}\right.$-acetone, $\left.3: 1 \rightarrow 1: 1\right)$ to yield $1(571.5 \mathrm{mg})$ and a mixture. The mixture was further purified by semipreparative HPLC [Agilent Zorbax SB-C 18 column, MeCN- $\mathrm{H}_{2} \mathrm{O}$ (containing 0.05\% TFA), 28:72, $2 \mathrm{~mL} / \mathrm{min}$ ] to yield $\mathbf{9}\left(5.9 \mathrm{mg}, t_{\mathrm{R}}=13.042 \mathrm{~min}\right), \mathbf{1 0}(5.8 \mathrm{mg}$, $\left.t_{\mathrm{R}}=17.294 \mathrm{~min}\right)$, and 11 (2.6 $\left.\mathrm{mg}, t_{\mathrm{R}}=19.588 \mathrm{~min}\right) . \mathrm{B} 3-2$ ( $90 \% \mathrm{MeOH}-$ eluted portion) was separated by Sephadex LH-20 column chromatography $(\mathrm{MeOH})$ and semipreparative HPLC (Welch Ultimate AQ- $\mathrm{C}_{18}$ column, $\mathrm{MeOH}-\mathrm{H}_{2} \mathrm{O}$, $87: 13,1 \mathrm{~mL} / \mathrm{min})$ to yield $4\left(3.5 \mathrm{mg}, t_{\mathrm{R}}=42.732 \mathrm{~min}\right)$ and 3 (2.0 $\mathrm{mg}, t_{\mathrm{R}}=64.797 \mathrm{~min}$ ).

Fraction B4 was separated on an $\mathrm{RP}^{-\mathrm{C}_{18}}$ silica gel column eluted with $\mathrm{MeOH}-\mathrm{H}_{2} \mathrm{O}(10 \% \rightarrow 100 \%)$ to yield three main fractions. The $10 \% \mathrm{MeOH}$-eluted portion was purified by silica gel column chromatography $\left(\mathrm{CH}_{2} \mathrm{Cl}_{2}-\mathrm{MeOH}\right.$, 30:1 and 20:1) and Sephadex LH-20 column chromatography $(\mathrm{MeOH})$ to yield $5(1.1 \mathrm{~g})$ recrystallized from $\mathrm{MeOH}$. The $15 \% \mathrm{MeOH}$-eluted portion was isolated by silica gel column chromatography $\left(\mathrm{CH}_{2} \mathrm{Cl}_{2}-\mathrm{MeOH}, 30: 1\right.$ and 20:1) and Sephadex LH-20 column chromatography (MeOH) and further purified by semipreparative HPLC (Welch Ultimate AQ- $\mathrm{C}_{18}$ column, $\mathrm{MeOH}-\mathrm{H}_{2} \mathrm{O}, 15: 85,1 \mathrm{~mL} / \mathrm{min}$ ) to yield $12\left(6.4 \mathrm{mg}, t_{\mathrm{R}}=36.095 \mathrm{~min}\right)$. The $20 \% \mathrm{MeOH}$-eluted portion was isolated by Sephadex LH-20 column chromatography $(\mathrm{MeOH})$ and semipreparative HPLC (Agilent Zorbax SB- ${ }_{18}$ column, $\mathrm{MeOH}-\mathrm{H}_{2} \mathrm{O}, 15: 85,2 \mathrm{~mL} / \mathrm{min}$ ) to yield 7 (1.6 $\mathrm{mg}, t_{\mathrm{R}}=22.265 \mathrm{~min}$ ).

\subsection{Spectroscopic Data of Compounds}

\subsubsection{Cuscutaroside A (1)}

White powder; $[\alpha]_{\mathrm{D}}^{21}-46.2(c=0.19, \mathrm{MeOH})$; UV $(\mathrm{MeOH})$ $\lambda_{\max }(\log \varepsilon) 311$ (4.42), 227 (4.11), 200 (4.49) nm; ${ }^{1} \mathrm{H}$ NMR and ${ }^{13} \mathrm{C}$ NMR data see Table 1; ESIMS (negative) $\mathrm{m} / \mathrm{z} 433$ [M-H] $]^{-}, 867$ [2 M-H]'; HRESIMS (positive) $\mathrm{m} / \mathrm{z} 457.1106$ $[\mathrm{M}+\mathrm{Na}]^{+}$(calcd for $\mathrm{C}_{21} \mathrm{H}_{22} \mathrm{NaO}_{10}, 457.1111$ ).

\subsubsection{Cuscutaroside B (2)}

Colorless solid; $[\alpha]_{\mathrm{D}}^{21}-77.6(c=0.11, \mathrm{MeOH})$; UV $(\mathrm{MeOH})$ $\lambda_{\max }(\log \varepsilon) 285$ (3.54), 198 (4.22) nm; ECD (c 0.011, $\mathrm{MeOH}) \lambda_{\max }(\Delta \varepsilon) 281(-0.91), 199(-4.06) \mathrm{nm} ;{ }^{1} \mathrm{H}$ NMR and ${ }^{13} \mathrm{C}$ NMR data see Table 1; ESIMS (positive) $\mathrm{m} / z .395$ 
$[\mathrm{M}+\mathrm{Na}]^{+}, 767[2 \mathrm{M}+\mathrm{Na}]^{+}$; HRESIMS (positive) $\mathrm{m} / \mathrm{z}$ $395.1313[\mathrm{M}+\mathrm{Na}]^{+}$(calcd for $\mathrm{C}_{17} \mathrm{H}_{24} \mathrm{NaO}_{9}, 395.1318$ ).

\subsubsection{7 $\beta$-Methoxy- $\beta$-sitosterol 3-O- $\beta$-glucopyranoside (3)}

Colorless solid; $[\alpha]_{\mathrm{D}}^{21}-25.2(c=0.11, \mathrm{MeOH}) ;{ }^{1} \mathrm{H}$ NMR and ${ }^{13} \mathrm{C}$ NMR data see Table 2; ESIMS (positive) $\mathrm{m} / z 629$ $[\mathrm{M}+\mathrm{Na}]^{+}$; HRESIMS (positive) $\mathrm{m} / z 629.4388[\mathrm{M}+\mathrm{Na}]^{+}$ (calcd for $\mathrm{C}_{36} \mathrm{H}_{62} \mathrm{NaO}_{7}, 629.4393$ ).

\subsection{Acidic Hydrolysis of Compound 1}

Compound 1 (20.0 mg, $0.046 \mathrm{mM}$ ) was dissolved in $15 \mathrm{~mL}$ of $6 \%$ aq. $\mathrm{HCl}$ and hydrolyzed under reflux $(5 \mathrm{~h})$ at $90{ }^{\circ} \mathrm{C}$. Then, the acidic solution was evaporated in vacuo to dryness and separated by silica gel column chromatography eluted with $\mathrm{CHCl}_{3}-\mathrm{MeOH}(10: 1)$ to yield D-glucopyranose (5.5 mg, $0.031 \mathrm{mM}, 67 \%$ yield), which was identified based on its ${ }^{1} \mathrm{H}$ NMR spectrum and optical rotation value of $[\alpha]_{\mathrm{D}}^{20}+40.0\left(c 0.09, \mathrm{H}_{2} \mathrm{O}\right)[26]$.

\subsection{Acetylation of Compound 1}

Compound 1 ( $20.6 \mathrm{mg}, 0.048 \mathrm{mmol}$ ) was dissolved in 500 $\mu \mathrm{L}$ of pyridine and $500 \mu \mathrm{L}$ of acetic anhydride and stirred for $24 \mathrm{~h}$ at room temperature. Then, water $(2 \mathrm{~mL})$ was added to the reaction mixture, followed by extraction with ethyl acetate $(4 \mathrm{~mL})$. The upper layer was dried under reduced pressure and purified by silica gel column chromatography (petroleum ether-EtOAc, 10:1) to yield 1a $(20.4 \mathrm{mg}$, $0.034 \mathrm{mmol}, 71 \%$ yield). White solid; ${ }^{1} \mathrm{H}$ NMR $(500 \mathrm{MHz}$, $\left.\mathrm{CDCl}_{3}\right) \delta 7.68\left(1 \mathrm{H}, \mathrm{d}, J=16.0 \mathrm{~Hz}, \mathrm{H}-7^{\prime \prime}\right), 7.61(2 \mathrm{H}, \mathrm{br} \mathrm{d}$, $J=8.6 \mathrm{~Hz}, \mathrm{H}-2^{\prime \prime}$ and $\left.\mathrm{H}-6^{\prime \prime}\right), 7.12\left(2 \mathrm{H}, \mathrm{br} \mathrm{d}, J=8.6 \mathrm{~Hz}, \mathrm{H}-3^{\prime \prime}\right.$ and $\left.\mathrm{H}-5^{\prime \prime}\right), 6.46\left(1 \mathrm{H}, \mathrm{d}, J=16.0 \mathrm{~Hz}, \mathrm{H}-8^{\prime \prime}\right), 5.80(1 \mathrm{H}, \mathrm{br}$ s, H-5), $5.63(1 \mathrm{H}, \mathrm{d}, J=2.2 \mathrm{~Hz}, \mathrm{H}-3), 5.28(2 \mathrm{H}, \mathrm{m}), 5.16$ $(2 \mathrm{H}, \mathrm{m}), 4.37(1 \mathrm{H}, \mathrm{dd}, J=12.6,2.3 \mathrm{~Hz}, \mathrm{H}-6 \mathrm{\prime}$ a $), 4.27(1 \mathrm{H}$, dd, $\left.J=12.6,6.2 \mathrm{~Hz}, \mathrm{H}-6^{\prime} \mathrm{b}\right), 3.94\left(1 \mathrm{H}, \mathrm{m}, \mathrm{H}-5^{\prime}\right), 2.30(3 \mathrm{H}$, s), $2.19(3 \mathrm{H}, \mathrm{s}), 2.06(3 \mathrm{H}, \mathrm{s}), 2.06(3 \mathrm{H}, \mathrm{s}), 2.03(3 \mathrm{H}, \mathrm{s})$; ${ }^{13} \mathrm{C} \mathrm{NMR}\left(125 \mathrm{MHz}, \mathrm{CDCl}_{3}\right) \delta 170.2,169.5,169.3,169.2$, $168.1,166.4,164.4,163.3,152.4,145.2,132.0,130.5$, 129.7, 122.3, 117.1, 100.1, 96.9, 91.4, 73.0, 72.6, 70.7, 68.2, 62.1, 21.3, 20.7, 20.7, 20.7, 20.0; ESIMS (positive) $\mathrm{m} / z 625$ $[\mathrm{M}+\mathrm{Na}]^{+}$; HRESIMS (positive) $\mathrm{m} / z 625.1528[\mathrm{M}+\mathrm{Na}]^{+}$ (calcd for $\mathrm{C}_{29} \mathrm{H}_{30} \mathrm{NaO}_{14}, 625.1533$ ).

\subsection{In vitro Porcine Pancreatic Lipase Inhibition Assay}

For lipase inhibition tests of each compound, porcine pancreatic lipase was used. $p$-Nitrophenyl butyrate ( $p$-NPB) was used as the substrate. First, $5 \mu \mathrm{L}$ of the lipase solution (40 U/ $\mathrm{mL})$ in Tris- $\mathrm{HCl}$ buffer (100 mM Tris- $\mathrm{HCl}, 5 \mathrm{mM} \mathrm{CaCl}_{2}$;
$\mathrm{pH}$ 7.0) was added to a 96-well microtiter plate. Each compound in $1 \mu \mathrm{L}$ of DMSO and $184 \mu \mathrm{L}$ of Tris- $\mathrm{HCl}$ buffer were added and mixed with the enzyme buffer to start the reaction. After incubation at $37^{\circ} \mathrm{C}$ for $15 \mathrm{~min}, 10 \mu \mathrm{L}$ of the substrate solution $(10 \mathrm{mM} p$-NPB in dimethyl formamide) was added. The enzymatic reaction was carried out for $15 \mathrm{~min}$ at $37^{\circ} \mathrm{C}$. The hydrolysis of $p$-NPB to $p$-nitrophenol was monitored at $400 \mathrm{~nm}$ using a spectrophotometer [27].

\subsection{In Vitro Platelet Aggregation Assay}

The inhibitory effects of the compounds against rabbit platelet aggregation induced by thrombin $(1 \mathrm{U} / \mathrm{mL}), \operatorname{PAF}(0.4 \mu \mathrm{g} /$ $\mathrm{mL})$, AA $(0.5 \mathrm{mM})$, or collagen $(4 \mu \mathrm{g} / \mathrm{mL})$ were evaluated according to published methods [28-30].

\section{Conclusion}

Three new and 12 known compounds were isolated from the whole plants of Cuscuta reflexa (Convolvulaceae) collected from Myanmar. $7 \beta$-Methoxy- $\beta$-sitosterol 3-O- $\beta$ glucopyranoside (3) showed weak PPL inhibitory activity $\left(\mathrm{IC}_{50}=67.2 \pm 1.7 \mu \mathrm{g} / \mathrm{mL}\right)$. Cuscutaroside A (1), its acetylated derivative (1a), and scrophenoside B (8) showed weak inhibitory activity against rabbit platelet aggregation induced by collagen $(4 \mu \mathrm{g} / \mathrm{mL})$ with $\mathrm{IC}_{50}$ values of $291.4 \pm 47.9 \mu \mathrm{g} /$ $\mathrm{mL}, 63.8 \pm 4.4 \mu \mathrm{g} / \mathrm{mL}$, and $180.5 \pm 6.7 \mu \mathrm{g} / \mathrm{mL}$, respectively. Compound 1a also showed inhibitory activity against rabbit platelet aggregation induced by $\mathrm{AA}(0.5 \mathrm{mM})$ with an $\mathrm{IC}_{50}$ value of $72.6 \pm 10.5 \mu \mathrm{g} / \mathrm{mL}$. These results support previous findings in pharmacological studies related to the traditional uses of the plant.

Acknowledgements This study was supported by the Southeast Asia Biodiversity Research Institute, Chinese Academy of Sciences (Grant Nos. 2015CASEABRIRG001 and Y4ZK111B01), the International Partnership Program of Chinese Academy of Sciences (Grant No. 153631KYSB20160004), the National Natural Science Foundation of China (31960480), the Joint Special Project of Local Undergraduate Universities in Yunnan Province, China (Grant No. 2018FH001-024), and the Second Tibetan Plateau Scientific Expedition and Research (STEP) Program of Ministry of Science and Technology of the People's Republic of China (Grant No. 2019QZKK0502).

\section{Compliance with Ethical Standards}

Conflicts of interest The authors declare that there are no conflicts of interest associated with this work.

Open Access This article is licensed under a Creative Commons Attribution 4.0 International License, which permits use, sharing, adaptation, distribution and reproduction in any medium or format, as long as you give appropriate credit to the original author(s) and the source, provide a link to the Creative Commons licence, and indicate if changes 
were made. The images or other third party material in this article are included in the article's Creative Commons licence, unless indicated otherwise in a credit line to the material. If material is not included in the article's Creative Commons licence and your intended use is not permitted by statutory regulation or exceeds the permitted use, you will need to obtain permission directly from the copyright holder. To view a copy of this licence, visit http://creativecommons.org/licenses/by/4.0/.

\section{References}

1. R.C. Fang, G. Staples, Convolvulaceae, in Flora of China, ed. by Z.Y. Wu, P.H. Raven, D.Y. Hong (Science Press, Beijing, 1995), pp. 271-325

2. Health Bureau of Lisu Autonomous Prefecture of Nujiang in Yunnan, Traditional Chinese Medicines in Nujiang (Yunnan Science \& Technology Press, Kunming, 1991), p. 263

3. R.A. DeFilipps, G.A. Krupnick, PhytoKeys 102, 1-341 (2018)

4. S. Noureen, S. Noreen, S.A. Ghumman, F. Batool, S.N.A. Bukhari, Iran. J. Basic Med. Sci. 22, 1225-1252 (2019)

5. N. Verma, R.K. Yadav, Plant Arch. 18, 1938-1942 (2018)

6. A. Ahmad, S. Tandon, T.D. Xuan, Z. Nooreen, Biomed. Pharmacother. 92, 772-795 (2017)

7. M.A. Versiani, A. Kanwal, S. Faizi, A.D. Farooq, Chem. Nat. Compd. 53, 915-922 (2017)

8. A. Poudel, S.G. Kim, Y.K. Kim, Y.S. Lee, G.W. Lee, B.S. Min, H.J. Jung, Nat. Prod. Sci. 17, 123-129 (2011)

9. S.L. Ong, S.H. Mah, H.Y. Lai, J. Pharmaceut. 2016, Article ID 8764274 (2016)

10. N. Tuvignon, A. Abousalham, F. Tocques, J. De Caro, A. De Caro, R. Laugier, F. Carrière, Anal. Biochem. 383, 289-295 (2008)

11. A.K. Azad, F.R. Laboni, H. Rashid, S. Ferdous, S.S. Rashid, N. Kamal, Z.K. Labu, M.S. Islam, Z.I. Sarker, Nat. Prod. Res. 34, 2394-2397 (2020)

12. S. Gafner, J.L. Wolfender, K. Hostettmann, H. Stoeckli-Evans, S. Mavi, Helv. Chim. Acta 81, 2062-2071 (1998)
13. S. Cai, A.L. Risinger, C.L. Petersen, T. Grkovic, B.R. O'Keefe, S.L. Mooberry, R.H. Cichewicz, J. Nat. Prod. 82, 928-936 (2019)

14. J.M. Fang, K.C. Wang, Y.S. Cheng, Phytochemistry 30, 3383$3387(1991)$

15. X.H. Li, L.D. Lin, P. Wu, M.F. Liu, X.Y. Wei, J. Trop. Subtrop. Bot. 15, 35-39 (2007)

16. N. Chaurasia, M. Wichtl, J. Nat. Prod. 50, 881-885 (1987)

17. G.R. Pettit, A. Numata, G.M. Cragg, D.L. Herald, T. Takada, C. Iwamoto, R. Riesen, J.M. Schmidt, D.L. Doubek, A. Goswami, J. Nat. Prod. 63, 72-78 (2000)

18. H.Y. Ding, H.C. Lin, C.M. Teng, Y.C. Wu, J. Chin. Chem. Soc. 47, 381-388 (2000)

19. Y. Pei, Z.D. Yang, J. Sheng, Chem. Nat. Compd. 50, 957-958 (2014)

20. S.X. Huang, X. Liao, Q.J. Nie, L.S. Ding, S.L. Peng, Helv. Chim. Acta 87, 598-604 (2004)

21. C.I. Tamayose, E.A. dos Santos, N. Roque, L.V. Costa-Lotufo, M.J.P. Ferreira, Chem. Biodivers. 16, e1900093 (2019)

22. Y. Yamano, M. Ito, Chem. Pharm. Bull. 53, 541-546 (2005)

23. Q. Luo, X. Cheng, Y.M. Yan, Y.X. Cheng, Nat. Prod. Res. Dev. 25(1311-1314), 1351 (2013)

24. S.H. Huang, J.R. Chen, F.Y. Tsai, Molecules 15, 315-330 (2010)

25. C.F. Zhang, Z.J. Zhang, M. Zhang, Z.T. Wang, Chin. Pharm. J. 41, 94-96 (2006)

26. Y.H. Wang, J.H. Wang, H.P. He, H. Zhou, X.W. Yang, C.S. Li, X.J. Hao, J. Asian Nat. Prod. Res. 10, 25-31 (2008)

27. J.M. Nicaud, C. Madzak, P. van den Broek, C. Gysler, P. Duboc, P. Niederberger, C. Gaillardin, FEMS Yeast Res. 2, 371-379 (2002)

28. L.J. Küster, J. Filep, J.C. Frölich, Thromb. Res. 43, 425-433 (1986)

29. M. Wu, D. Wen, N. Gao, C. Xiao, L. Yang, L. Xu, W. Lian, W. Peng, J. Jiang, J. Zhao, Eur. J. Med. Chem. 92, 257-269 (2015)

30. M.Y. Xia, J. Yang, P.H. Zhang, X.N. Li, J.F. Luo, C.L. Long, Y.H. Wang, Nat. Prod. Bioprospect. 8, 419-430 (2018) 